\title{
Review
}

\section{LUBAC-mediated linear ubiquitination: a crucial regulator of immune signaling}

\author{
By Kazuhiro IWAI ${ }^{* 1, \dagger}$
}

(Edited by Shigekazu NAGATA, M.J.A.)

\begin{abstract}
Ubiquitination is a reversible post-translational modification in which ubiquitin chains are conjugated to target proteins to modulate protein function. The type of ubiquitin chain determines the mode of protein regulation. It has been shown that ubiquitin chains are formed via one of seven Lys residues in ubiquitin, and several types of ubiquitin chains are found in cells. We identified a new type of linear ubiquitin chain linked through the N-terminal Met of ubiquitin and assembled by the linear ubiquitin chain assembly complex (LUBAC), which is specific for linear chains. The discovery of linear ubiquitin chains and LUBAC is considered as a paradigm shift in ubiquitin research because linear ubiquitination is exclusive to animals, despite the existence of ubiquitination throughout eukaryotic kingdoms. Linear ubiquitination plays a critical role in immune signaling and cell death regulation. Dysregulation of LUBAC-mediated linear ubiquitination underlies various human diseases, including autoinflammation, autoimmunity, infection, and malignant tumors. This review summarizes the current status of linear ubiquitination research.
\end{abstract}

Keywords: linear ubiquitination, LUBAC, NF- $\kappa$ B, cell death, cancer, inflammatory diseases

\footnotetext{
*1 Department of Molecular and Cellular Physiology, Graduate School of Medicine, Kyoto University, Kyoto, Japan.

$\dagger$ Correspondence should be addressed: K. Iwai, Department of Molecular and Cellular Physiology, Graduate School of Medicine, Kyoto University, Yoshida-konoe-cho, Sakyo-ku, Kyoto 606-8501, Japan (e-mail: kiwai@mcp.med.kyoto-u.ac.jp).

Abbreviations: K: lysine; LUBAC: linear ubiquitin chain assembly complex; IRP2: iron regulatory protein 2; HOIL-1: hemeoxidized IRP2 ubiquitin ligase 1; HOIP: HOIL-1L interacting protein; SHARPIN: SHANK-associated RH domain-interacting protein; UBA: ubiquitin-associated; RBR: RING-in-betweenRING; LDD: linear ubiquitin chain-determining domain; LTM: LUBAC-tethering motif; NEMO: NF- $\kappa$ B essential modifier; DUB deubiquitinating enzyme; OTULIN: OTU deubiquitinase with linear linkage specificity; CYLD: cylindromatosis; PUB: PNGase/ ubiquitin-associated; SPATA2: spermatogenesis associated 2; UBAN: ubiquitin binding in ABIN and NEMO; NZF: Npl4 zinc finger; ZF: zinc finger; NF- $\kappa \mathrm{B}$ : nuclear factor- $\kappa \mathrm{B}$; TNFR1: TNFreceptor 1; RIPK1: receptor interacting serine/threonine-protein kinase 1; cIAP: cellular inhibitor of apoptosis protein; I $\kappa \mathrm{B}$ : inhibitor of $\kappa \mathrm{B}$; IKK: $\mathrm{I} \kappa \mathrm{B}$ kinase complex; MyD88: myeloid differentiation primary response 88; TLR: Toll-like receptor; IRAK: interleukin-1 receptor-associated kinase; TCR: T-cell receptor; BCR: B-cell receptor; FADD: FAS-associated death domain protein; cFLIP: FLICE (FADD-like IL-1 $\beta$-converting enzyme)-inhibitory protein; ASC: apoptosis-associated speckled protein containing a CARD; IFN: interferon; cpdm: chronic proliferative dermatitis in mice; ORAS: OTULIN-related autoinflammatory syndrome; SNPs: single-nucleotide polymorphisms; ABIN1: A20-binding inhibitor of NF- $\kappa B 1$; ABC-DLBCL: B-celllike diffuse large B-cell lymphoma.
}

\section{Introduction}

Proteins are vital for cellular functions and can form molecules of diverse shapes and sizes. The size and shape of molecules are critical determinants of their function. Proteins are not always functional and they exert their function in a timely manner. Covalent post-translational modifications modulate protein function in a timely and selective manner. There are many types of post-translational modifications. ${ }^{1)}$ Ubiquitin is a highly conserved and unique post-translational modifier that is conjugated to target proteins as monoubiquitin or as a ubiquitin chain, which is a polymer of ubiquitin moieties, thereby regulating protein functions. ${ }^{2)}$

The ubiquitin conjugation system was identified as part of the energy-dependent protein degradation pathway. ${ }^{3)}$ In this pathway, ubiquitin chains function as recognition tags for the $26 \mathrm{~S}$ proteasome, and ubiquitinated proteins are destined for degradation in a timely and selective manner. ${ }^{2)}$ Ubiquitin research has flourished in close association with proteolysis, and three scientists were awarded the Nobel Prize in Chemistry for the discovery of ubiquitin-mediated protein degradation in 2004. However, the research 
field of ubiquitin has expanded in different directions in the "post-Nobel era", and the system is currently recognized as one of the most sophisticated reversible post-translational modification systems regulating protein function in many ways. ${ }^{4}$

Conjugation of ubiquitin chains was originally thought to target proteins for proteolysis, ${ }^{2)}$ and lysine (K) 48-linked ubiquitin chains were described as degradation tags. ${ }^{5)}$ Further research revealed the presence of multiple types of ubiquitin chains in cells with different roles in the regulation of conjugated proteins, because each type of ubiquitin chain can be decoded by specific binding proteins. ${ }^{4)}$ Non-degradation-related roles of ubiquitin started to be analyzed when K63-mediated ubiquitin chains were shown to be involved in DNA repair and signaling without functioning as a degradation signal. ${ }^{6)}$ Ubiquitin has seven K residues (K6, K11, K27, K29, K33, K48, and $\mathrm{K} 63$ ), and mass spectrometric analyses indicate that ubiquitin linkages can be formed via any of the seven $\mathrm{K}$ residues but not via other residues. ${ }^{7}$ In these circumstances, we discovered a new type of ubiquitin chain termed the linear ubiquitin chain or M1 chain that is generated by conjugation of the C-terminal carboxyl group of a donor ubiquitin and the $\alpha$-amino group of the N-terminal methionine of an acceptor ubiquitin. ${ }^{8)}$ We also identified the linear ubiquitin chain assembly complex (LUBAC) as the specific ligase catalyzing the assembly of linear chains. ${ }^{8)}$ This review article describes the molecules involved in the metabolism and function of linear ubiquitin chains and the current status of linear ubiquitin research.

\section{Molecules mediating the synthesis and cleavage of M1-linked linear ubiquitin chains}

Post-translational modifications including ubiquitination regulate cellular functions in a timely and selective manner through the coordinated actions of enzymes that mediate synthesis (writers), digestion (erasers), and recognition (readers).

a) Enzymes catalyzing the synthesis of linear ubiquitin chains. Ubiquitin modification is catalyzed by the repetitive function of three enzymes: E1 (ubiquitin activating enzyme), E2 (ubiquitin conjugating enzyme), and E3 (ubiquitin ligase). ${ }^{2)}$ LUBAC is the only E3 enzyme complex identified to date that catalyzes the synthesis of linear ubiquitin chains. $^{2)}$ LUBAC is composed of three subunits: a large isoform of heme-oxidized iron regulatory protein 2 (IRP2) ubiquitin ligase 1 (HOIL-1L), HOIL-1L interacting protein (HOIP), and SHANK-associated $\mathrm{RH}$ domain-interacting protein (SHARPIN)

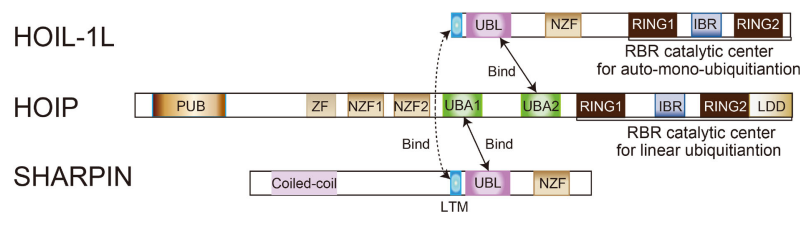

Fig. 1. (Color online) The LUBAC ubiquitin ligase. Schematic representation of the LUBAC ubiquitin ligase complex. LUBAC consists of HOIL-1L, HOIP, and SHARPIN. HOIP is the catalytic center for linear ubiquitination. UBL, ubiquitin-like; NZF, NPl4-type zinc finger; RING, really interesting new gene; IBR, in-between RING; RBR, RING-in-between-RING; ZF, zinc finger; UBA, ubiquitin-associated domain; LDD, linear ubiquitin chain-determining domain; PUB, peptide N-glycosidase (PNGase)/ubiquitin-associated; LTM, LUBAC-tethering motif.

(Fig. 1). ${ }^{8)-11)}$ The RING-in-between-RING (RBR) domain of HOIP is the catalytic center for linear ubiquitination. ${ }^{8)}$ HOIP interacts with the E2 carrying ubiquitin at RING1 and transfers ubiquitin from the E2 to a specific Cys residue (Cys885) in RING2. It then catalyzes the formation of a peptide bond between the C-terminal carboxyl group of ubiquitin on Cys885 and the $\alpha$-amino group of the acceptor ubiquitin, which is recognized by the linear ubiquitin chain-determining domain (LDD) of HOIP. ${ }^{12), 13)}$ The stability of the LUBAC complex is determined by the interactions between its three subunits. ${ }^{14), 15)}$ HOIP interacts with the ubiquitin-like domain of the other two subunits via the ubiquitin-associated domain. ${ }^{14)}$ The interaction between HOIL-1L and SHARPIN is unique because the LUBAC-tethering motif (LTM) of the two proteins folds into a single globular domain; LTM-mediated dimerization is critical for stable LUBAC formation, and mutation of the HOIL-1L LTM drastically destabilizes the complex. ${ }^{15)}$ The three subunits form a complex with a stoichiometry of 1:1:1; however, the exact number of subunits in the LUBAC complex remains undefined, because gel filtration analyses showed that LUBAC forms a $600 \mathrm{KDa}$ complex. ${ }^{8)}$ HOIL-1L also has an RBR E3 center. ${ }^{8)}$ Although it has been suggested that it facilitates linear ubiquitination of $\mathrm{NF}-\kappa \mathrm{B}$ essential modulator (NEMO), a substrate of LUBAC, ${ }^{16)}$ the HOIL-1L E3 is now known to catalyze the formation of an oxy-ester bond between the C-terminal carboxyl group of ubiquitin and the hydroxyl groups of Ser and/or Thr residues of LUBAC subunits and substrates. ${ }^{17)}$ However, we showed that HOIL-1L monoubiquitinates LUBAC subunits via iso-peptide linkages to $\mathrm{K}$ residues, which plays a crucial role in the regulation of LUBAC, 
although the HOIL-1L E3 catalyzes oxy-ester ubiquitination (as described in detail below). ${ }^{18)}$

b) Enzymes catalyzing the digestion of linear ubiquitin chains. Ubiquitin chains conjugated to proteins are cleaved by deubiquitinating enzymes (DUBs). Approximately 90 DUBs have been identified in humans, and some DUBs selectively digest specific inter-ubiquitin linkages. Two DUBs, OTU deubiquitinase with linear linkage specificity (OTULIN) and cylindromatosis (CYLD), cleave linear chains selectively; however, CYLD cleaves K63-linked ubiquitin chains in addition to linear chains. ${ }^{19)}$ Although both DUBs interact with LUBAC via the PNGase/ubiquitin-associated (PUB) domain of HOIP [CYLD interacts with HOIP via spermatogenesis associated 2 (SPATA2)], the two DUBs behave differently. ${ }^{20)-23)}$ CYLD was identified as a causative gene product of human cylindromatosis. ${ }^{24)}$ Although CYLD was described as a DUB specific for K63 chains involved in the activation of NF- $\kappa \mathrm{B},{ }^{25)}$ it cleaves linear chains more efficiently than K63 chains. Ectopic expression of CYLD suppresses LUBAC-mediated NF- $\kappa$ B activation; however, loss of CYLD does not have a substantial effect on the amount of linear ubiquitination. Consistent with this, its involvement in the regulation of linear ubiquitination is not as clear as that of OTULIN. In contrast to CYLD, OTULIN plays an important role in regulating linear ubiquitin chains in cells, and the loss of OTULIN DUB activity markedly increases the amount of linear ubiquitin chains. ${ }^{26}$ ) The linear ubiquitin chains in OTULIN null cells are mostly conjugated to LUBAC subunits, which is called auto-ubiquitination. Auto-linear ubiquitination attenuates LUBAC function, suggesting that OTULIN is a unique DUB that does not counteract but rather maintains the function of the LUBAC ubiquitin ligase by trimming ubiquitin chains (as described in detail below). ${ }^{27}$ )

c) Linear ubiquitin-binding proteins. Posttranslational modifications are recognized by binding proteins called "readers". Because the type of ubiquitin chain determines the mode of protein regulation, ubiquitin linkages are decoded by specific binding proteins that transmit signals to mediate the function of each chain. In addition to ubiquitin chains composed of homologous linkages, heterotypic ubiquitin chains contain different linkage types. ${ }^{28), 29)}$ Mixed or hybrid chains contain one linkage type that is extended by a second linkage type, forming an unbranched structure. Hybrid chains composed of linear and K63 chains have been reported, in which the proximal part of the chain consists of K63 linkages and the distal part is composed of linear linkages. ${ }^{30)}$ In branched chains, a ubiquitin molecule in the chain may be ubiquitinated at multiple $\mathrm{K}$ residues. Branched chains including linear linkages have not been reported to date. Because all homogeneous and heterogenous chains can be formed by eight different inter-ubiquitin linkages using one of seven $K$ residues and one N-terminal Met of ubiquitin, even mixed and branched chains can be decoded by the coordinated action of ubiquitinbinding proteins that recognize the specific linkages within the di-ubiquitin. Several proteins that specifically recognize linear di-ubiquitin have been reported, ${ }^{31)}$ including proteins that conduct ubiquitin binding in the ABIN and NEMO (UBAN) domain, which binds to linear di-ubiquitin specifically. Of five proteins containing the UBAN domain, four have been shown to bind to linear di-ubiquitin with high affinity. In addition to the UBAN proteins, HOIL-1L and A20 bind to linear ubiquitin chains via the NPL4 zinc finger (NZF) and zinc finger (ZF) 7 domains, respectively. ${ }^{32)-34)}$ Linear chains conjugated to substrate proteins by LUBAC are recognized by specific reader proteins, and their mechanism of action will be discussed later.

\section{Physiological functions of linear ubiquitination}

The role of linear ubiquitination, which has been studied extensively since its discovery, is mostly related to inflammatory responses.

a) NF- $\kappa \mathbf{B}$ activation. The most extensively studied function of linear ubiquitination is the activation of nuclear factor- $\kappa \mathrm{B}(\mathrm{NF}-\kappa \mathrm{B}) .{ }^{35), 36)} \mathrm{NF}$ $\kappa \mathrm{B}$ is a dimeric transcription factor involved in pleomorphic functions, including immune responses and cell proliferation, and it plays a central role in inflammatory processes. ${ }^{37)}$ LUBAC-mediated NF- $\kappa \mathrm{B}$ activation has been studied extensively in relation to TNF- $\alpha$ signaling (Fig. 2). Upon binding of TNF- $\alpha$ to TNF-receptor 1 (TNFR1), several signaling adaptor molecules, such as TNFR1-associated death domain. TNF-receptor associated factor 2, receptor interacting serine/threonine-protein kinase 1 (RIPK1), and cellular inhibitor of apoptosis proteins 1 and 2 (cIAP1/2), are recruited to TNFR1 to form complex I. In TNFR1 complex I, the cIAP ubiquitin ligases conjugate K63 and/or K11 chains to components of the complex. ${ }^{14)}$ LUBAC is recruited to complex I via recognition of K63 ubiquitin chains on the complex by the NZF domains of HOIP and SHARPIN. ${ }^{38), 39)}$ LUBAC also recognizes NEMO 


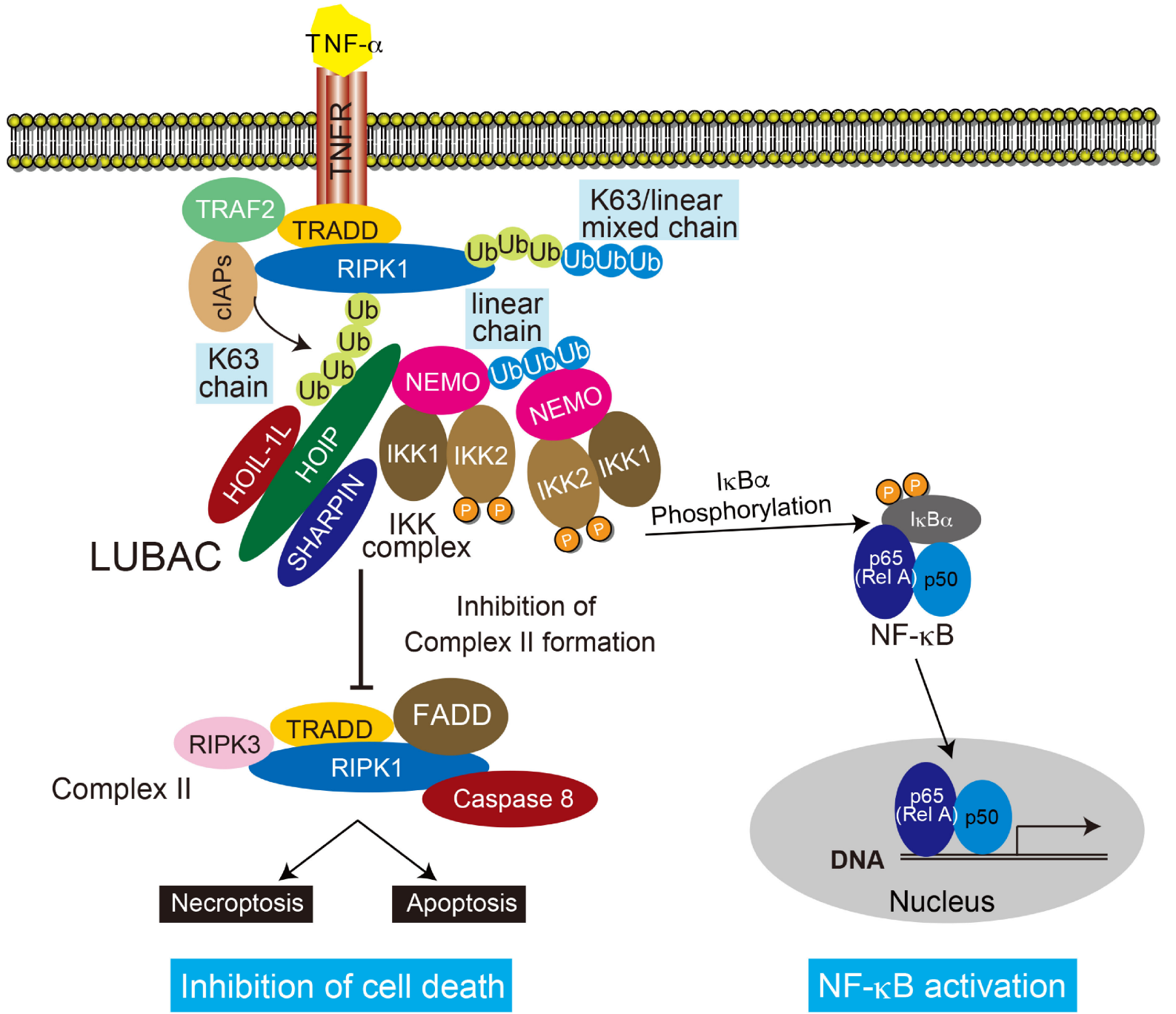

Fig. 2. (Color online) Physiological functions of LUBAC-mediated linear ubiquitination. LUBAC is recruited to the activated TNFreceptor complex via recognition of ubiquitin chains generated by cIAP1/2 and conjugates to linear-ubiquitinated NEMO. Linear chains conjugated to NEMO are recognized by the UBAN domain of NEMO in another IKK complex, which leads to activation of IKK2 by dimerization, autophosphorylation of IKK2, and the subsequent activation of NF- $\kappa$ B. Linear ubiquitination of components of the activated TNF-receptor complex decrease the production of death-inducible complex II containing RIPK1, RIPK3, FADD, and caspase-8, thereby suppressing death receptor-induced programmed cell death, including apoptosis and necroptosis.

through the HOIP NZF1 domain and conjugates linear ubiquitin to NEMO. ${ }^{38)} \mathrm{NEMO}$ is the regulatory component of the inhibitor of $\kappa \mathrm{B}(\mathrm{I} \kappa \mathrm{B})$ kinase complex (IKK), which is composed of IKK1, IKK2, and NEMO. Because the UBAN domain of NEMO interacts with linear di-ubiquitin with high affinity, ${ }^{40), 41)}$ the linear ubiquitin chain-conjugated NEMO is recognized by NEMO in another IKK complex, which leads to activation of IKK2 via dimerization and trans-autophosphorylation of the kinases in different IKK complexes. ${ }^{38)}$ Activated
IKK2 phosphorylates $\mathrm{I} \kappa \mathrm{B}$ to activate NF- $\kappa \mathrm{B}$. Loss of LUBAC suppresses NF- $\kappa$ B activation upon TNFR1 or CD40 stimulation, supporting the critical role of LUBAC-mediated linear ubiquitination in canonical NF- $\kappa$ B activation via the TNFR family. ${ }^{42), 43)}$ A reader of linear ubiquitin is also involved in the termination of $\mathrm{NF}-\kappa \mathrm{B}$ activation. The A20 deubiquitinase is induced by NF- $\kappa \mathrm{B}$ to suppress NF$\kappa \mathrm{B}$ activation. ${ }^{19), 44)}$ The DUB activity of A20 is not necessary for the suppression, whereas interaction with linear ubiquitin at the ZF7 domain of A20 is 
crucial for NF- $\kappa \mathrm{B}$ suppression; the precise mechanism underlying the suppression of NF- $\kappa \mathrm{B}$ activity remains to be elucidated. ${ }^{33), 34)}$ In addition to signaling via the TNF-receptor family, LUBAC-mediated linear ubiquitination is involved in NF- $\kappa \mathrm{B}$ activation in other immune signaling pathways. Signaling by nucleotidebinding oligomerization domain-containing protein 2 is characterized by the linear ubiquitination of RIPK2 by LUBAC upon recognition of pattern-associated molecular patterns by sensors, which leads to the activation of NF- $\kappa$ B. $\left.{ }^{45}\right)$ Myeloid differentiation primary response 88 (MyD88)-dependent NF- $\kappa \mathrm{B}$ activation involves signaling via Toll-like receptors (TLRs) and IL-1 receptors. In this process, LUBAC is recruited to the MyD88 activation complex (MyDDosome), which includes interleukin-1 receptor-associated kinase 1 (IRAK1), IRAK4, MyD88, and TRAF6, through recognition of K63 chains conjugated to the MyDDosome by TRAF6. ${ }^{30), 46)}$ Therefore, LUBAC functions in $\mathrm{NF}-\kappa \mathrm{B}$ activation by catalyzing the conjugation of linear chains to NEMO and components of the MyDDosome. ${ }^{11), 42)}$

LUBAC-mediated linear ubiquitination activity is dispensable for NF- $\kappa \mathrm{B}$ activation via antigen receptors of lymphocytes, although the LUBAC protein complex is necessary. ${ }^{42), 47), 48)}$ The complex composed of caspase recruitment domain-containing membrane-associated guanylate kinase protein-1, Bcell lymphoma/leukemia 10, and mucosa-associated lymphoid tissue lymphoma translocated gene 1 is involved in $\mathrm{NF}-\kappa \mathrm{B}$ activation mediated by T-cell receptor (TCR) and B-cell receptor (BCR) signaling. ${ }^{49)}$ Although linear ubiquitination of B-cell lymphoma/leukemia 10 by LUBAC is involved in $\mathrm{NF}-\kappa \mathrm{B}$ activation, ${ }^{50)}$ the ubiquitination activity of LUBAC is dispensable for both BCR- and TCRmediated NF- $\kappa \mathrm{B}$ activation, as indicated by the activation of $\mathrm{NF}-\kappa \mathrm{B}$ by B-cells expressing ligase defective HOIP in BCR signaling. ${ }^{42)}$ However, deletion or reduction of HOIP abolishes NF- $\kappa \mathrm{B}$ activation mediated by TCR and BCR, suggesting that the LUBAC protein complex is essential for antigen receptor-mediated $\mathrm{NF}-\kappa \mathrm{B}$ signaling. ${ }^{47), 48)}$

Thus, the mode of involvement of LUBAC in $\mathrm{NF}-\kappa \mathrm{B}$ activation is context dependent. For example, loss of linear ubiquitination activity of LUBAC suppresses TNF- $\alpha$-induced NF- $\kappa \mathrm{B}$ activation almost completely, whereas TCR-mediated NF- $\kappa \mathrm{B}$ activation is not affected. The precise roles of linear ubiquitination and LUBAC, including its role as a scaffold complex in NF- $\kappa \mathrm{B}$ activation, remain to be clarified. b) Suppression of programmed cell death. Another well-established function of LUBAC-mediated linear ubiquitination is protection from programmed cell death including apoptosis and necroptosis. ${ }^{51)}$ In addition to NF- $\kappa \mathrm{B}$ activation, TNF- $\alpha$ induces apoptosis and necroptosis. The two cell death mechanisms are triggered by conversion of TNFR signaling complex I to death-inducible complex II, which comprises RIPK1, RIPK3, FAS-associated death domain protein (FADD), cellular FADD-like IL-1 $\beta$-converting enzyme (FLICE)-inhibitory protein (cFLIP), and caspase- $8 .^{51)}$ Inhibition of complex II formation requires the linear ubiquitination activity of LUBAC, which decorates several proteins including RIPK1 and TNFR1 in the TNFR1 complex I with linear ubiquitin. ${ }^{52)}$ Although the precise mechanism underlying the conjugation of linear chains to the TNFR1 complex remains unclear, it is assumed that linear ubiquitin chains are added to ubiquitin chains, possibly K63 chains, conjugated to the components of complex I by cIAP ligases, because ubiquitin itself, which is recognized by HOIP LDD (Fig. 1), is the preferable target for HOIP E3. ${ }^{9}$ Indeed, K63/linear hybrid chains, ${ }^{30)}$ in which proximal and distal linkages are K63 and linear-mediated, respectively, are present in the activated TNFR1 complex. It has been speculated that linear ubiquitin chains on the TNFR1 complex maintain the interaction between LUBAC and the TNFR1 complex, thereby inhibiting the formation of complex II, ${ }^{14), 52)}$ although the reader proteins for linear chains involved in suppressing the conversion to complex II have not been identified. Evidence supports the involvement of LUBAC in the suppression of programmed cell death elicited by stimuli other than TNFR1: loss of TNFR1 cannot rescue the lethality caused by loss of HOIP, as demonstrated by increased cell death in mice. ${ }^{43)}$ In addition to programmed cell death via death receptors, LUBAC is involved in the protection of genotoxin-induced apoptosis by linear ubiquitination of NEMO for NF- $\kappa$ B activation. ${ }^{53), 54)}$

The ubiquitin system, including seven types of K-linked ubiquitin chains, is distributed throughout eukaryotic kingdoms. However, the linear ubiquitin chain emerged during the evolution of animals. A homolog of HOIP, but not of HOIL-1L and SHARPIN, is found in drosophila, in which linear chains are involved in stress responses. ${ }^{55)}$ However, homologs of LUBAC and OTULIN are detected in the genus ciona. ${ }^{56)}$ Molecular expansion of the cell death signaling machinery may have occurred in ascidians such as ciona, which shares many central 
participants of the cell death signaling pathway with vertebrates. ${ }^{57)}$ Thus, linear ubiquitination may have evolved to intricately regulate survival and the cell death pathway.

c) Other functions. In addition to NF- $\kappa \mathrm{B}$ activation and protection against cell death, linear ubiquitin chains exert other roles, three of which are described below.

c)-i) Inflammasome activation. The inflammasome is a high-molecular weight protein complex assembled in the cytosol of inflammatory cells. Upon recognition of pathogens or endogenous danger signals, activated sensor proteins oligomerize and recruit adaptor proteins, including apoptosis-associated speckled protein containing a CARD (ASC) and caspase-1. This leads to the activation of inflammatory cytokines, including IL- $1 \beta$ and IL-18, and induces a programmed cell death mechanism called pyroptosis. ${ }^{58)}$ It has been implied that LUBACmediated linear ubiquitination of ASC is necessary for inflammasome activation in macrophages. ${ }^{59)}$

c)-ii) Selective autophagy. The involvement of ubiquitin chains in selective autophagy is welldocumented, and linear chains play a role in selective autophagy. ${ }^{60}$ ) Optineurin, an autophagy adaptor, specifically interacts with linear chains through its UBAN motif. ${ }^{61)}$ Cellular components decorated with linear ubiquitin chains, including impaired mitochondria or intracellular pathogens, are eliminated via autophagy. ${ }^{62), 63)}$ A mutation in the UBAN motif of Optineurin that impairs ubiquitin recognition is a causative agent of amyotrophic lateral sclerosis. ${ }^{64), 65)}$ Optineurin is also involved in the clearance of damaged mitochondria via autophagy through the process called PINK1/ Parkin-mediated mitophagy. ${ }^{66)}$

c)-iii) Interferon signaling. Type I interferons (IFNs) play crucial roles in antiviral responses. Recognition of pattern-associated molecular patterns of viruses by various pattern recognition receptors, including TLRs and retinoic acid-inducible gene-Ilike receptors, induces IFN expression. ${ }^{67)}$ LUBACmediated linear ubiquitination is involved in IFN induction, and global attenuation of LUBAC function in mice enhances IFN expression. ${ }^{27)}$ It has been shown that LUBAC suppresses retinoic acid-inducible gene-I-mediated IFN induction, ${ }^{68)}$ whereas LUBAC activity is required for IFN induction by norovirus, which is triggered by another retinoic acid-inducible gene-I-like receptor: melanoma differentiation-associated gene $5 .^{69)}$ Thus, LUBAC is involved in IFN activation through different mecha- nisms in a context-dependent manner, and further analyses are necessary to clarify its roles.

\section{Pathology of LUBAC-mediated linear ubiquitination}

As expected from the roles LUBAC plays in crucial cellular functions including NF- $\kappa \mathrm{B}$ activation and cell death suppression, abnormal LUBAC function underlies several diseases.

a) Immune-related disorders.

a)-i) Autoinflammatory diseases. Attenuated linear ubiquitination causes autoinflammatory diseases with immunodeficiency. Autoinflammatory diseases, which are caused by defect(s) or dysregulation of the innate immune system, are characterized by recurrent or continuous inflammation and the lack of a primary pathogenic role for the adaptive immune system. ${ }^{70)}$ The involvement of linear ubiquitination in the disorder was first suggested in a study of mice. Sharpin was identified in 2007 as a causative gene of chronic proliferative dermatitis in mice (cpdm) after the discovery of a spontaneous mutant mouse strain exhibiting chronic skin inflammation and immunodeficiency in 1993. ${ }^{71), 72)}$ The identification of SHARPIN as a third component of LUBAC by our group clarified the pathogenesis of cpdm mice. ${ }^{9)-11)}$ Loss of HOIP, the catalytic subunit of LUBAC, causes embryonic lethality in mice, ${ }^{42), 43)}$ whereas cpdm mice are viable despite the loss of SHARPIN, an accessory subunit of LUBAC. Although deletion of SHARPIN in cpdm drastically reduces the amount of HOIP, a small amount of the LUBAC complex composed of HOIL-1L and HOIP remains. ${ }^{11)}$ Thus, loss of SHARPIN markedly reduces linear chain formation in cells, which leads to cpdm symptoms. Our identification that decreased LUBAC activity underlies autoinflammatory diseases with immunodeficiency led to the discovery of human diseases associated with attenuated LUBAC function. Mutations in the genes encoding LUBAC subunits, such as Hoil-1l (Rbck1), and Hoip (Rnf31), cause human immunodeficiency and autoinflammation; however, patients with mutations in SHARPIN have not been reported to date. ${ }^{73), 74)}$

OTULIN, a DUB that specifically cleaves linear ubiquitin chains, is associated with an autoinflammatory disease called OTULIN-related autoinflammatory syndrome (ORAS). ${ }^{26), 75)}$ When discovered, it had been suspected that loss of OTULIN increases the amount of linear ubiquitin chains in cells, which induces inflammatory responses by promoting NF$\kappa \mathrm{B}$ activation. ${ }^{76)}$ However, decreased OTULIN DUB 


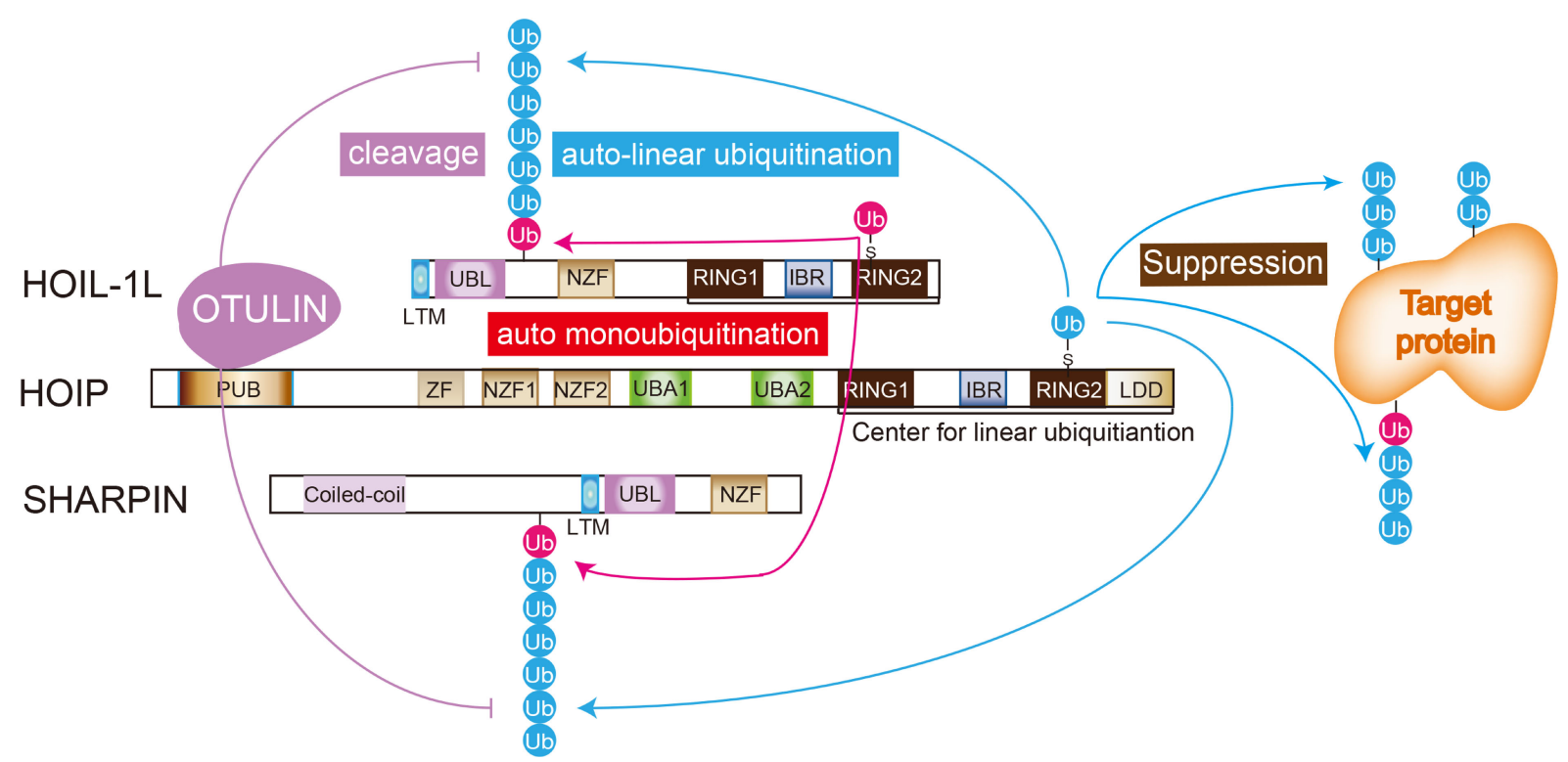

Fig. 3. (Color online) Regulation of the linear ubiquitination activity of LUBAC by the HOIL-1L E3 and OTULIN. The HOIL-1L E3 monoubiquitinates the three subunits of LUBAC including HOIL-1L. The HOIP E3 then conjugates linear chains onto LUBAC on monoubiquitin (auto-linear ubiquitination) to suppress LUBAC function. OTULIN trims linear chains conjugated onto LUBAC subunits. Thus, loss of the HOIL-1L E3 promotes LUBAC activity.

activity causes autoinflammatory disease by attenuating, rather than increasing, LUBAC functions. ${ }^{27)} \mathrm{In}$ general, erasers of post-translational modifications counteract the functions of writers by removing the modifications from target proteins; however, this is not the case with LUBAC and OTULIN. The unusual phenotypes associated with OTULIN mutations are underscored by intricate systems regulating the linear ubiquitination activity of HOIP mediated by the HOIL-1L E3 and OTULIN (Fig. 3). ${ }^{18)}$ LUBAC is a unique E3 because it possesses two ubiquitin ligase centers, one on HOIP for linear ubiquitination and one on HOIL-1L (Fig. 1). ${ }^{8)}$ LUBAC also interacts with OTULIN via the PUB domain of HOIP. ${ }^{20), 77), 78)}$ HOIL-1L in LUBAC displays two signals in immunoblotting, and loss of HOIL-1L ligase activity eliminates the slower migrating band; ${ }^{18}$ this led to the identification of the intricate regulatory mechanism of linear ubiquitination. The HOIL-1L E3 monoubiquitinates the three subunits of LUBAC including HOIL-1L. The HOIP E3 preferably recognizes ubiquitin at the LDD domain and conjugates ubiquitin onto the $\alpha$-amino group of the N-terminus of ubiquitin; ${ }^{13)}$ therefore, monoubiquitination of LUBAC subunits by HOIL-1L provides suitable substrates for the HOIP-mediated formation of linear chains on LUBAC (auto-linear ubiquitination). Indeed, loss of the HOIL-1L E3 suppresses auto-linear ubiquitination of LUBAC and promotes linear ubiquitination of target proteins. ${ }^{18)}$ Auto-linear ubiquitination of LUBAC by the HOIP E3 suppresses LUBAC function, although the precise underlying mechanism remains unknown. ${ }^{27)}$ OTULIN trims linear chains conjugated onto LUBAC subunits by interacting with HOIP, thereby maintaining LUBAC function. ${ }^{18), 27)}$ The functions of LUBAC are suppressed in ORAS patients because the auto-linear ubiquitination of LUBAC is increased by the inhibition of OTULIN catalytic activity.

a)-ii) Autoimmune diseases. Linear ubiquitination is also involved in autoimmune diseases. In autoimmune diseases, dysregulation of the immune system results in the activation of immune cells involved in acquired immunity to attack autoantigens. Single-nucleotide polymorphisms (SNPs) of the A20-binding inhibitor of NF- $\kappa B 1$ (ABIN1), also called TNIP1, are associated with an increased risk of developing systemic lupus erythematosus. ${ }^{79)}$ The ABIN1 protein has a UBAN domain that binds to linear ubiquitin chains with high affinity. ${ }^{80)}$ Loss of ABIN1 or the ubiquitin-binding activity of ABIN1 (ABIN1 D485N) in mice causes glomerulonephritis, which is characteristic of lupus nephritis with a high titer of pathogenic autoantibodies, including anti- 
nuclear antibody and anti-double strand DNA antibody. ${ }^{81,82)}$ The involvement of TLR7-MyD88IRAK4 signaling pathways in lupus nephritis has been suggested, ${ }^{83)}$ and lupus nephritis in ABIN1 D485N mice is ameliorated by IRAK4 inhibition. ${ }^{83)}$ Further analyses are necessary to clarify the roles of linear ubiquitination in autoimmune diseases.

b) Malignant tumors. LUBAC dysregulation is involved in oncogenesis. Rare germline SNPs of Hoip (Rnf31) are enriched in patients with B-celllike diffuse large B-cell lymphoma (ABC-DLBCL). ${ }^{84)}$ The SNPs induce the substitution of amino acids that increase the linear ubiquitination activity of LUBAC by promoting the interaction between HOIL-1L and HOIP. ${ }^{84)}$ Because constitutive activation of NF- $\kappa \mathrm{B}$ is the hallmark of ABC-DLBCL, ${ }^{85)}$ we examined the role of LUBAC-mediated linear ubiquitination in B-lymphoma. For this purpose, we generated mice overexpressing HOIP, the catalytic subunit of LUBAC, in B-cells. ${ }^{54)}$ Although increased LUBAC activity per se failed to induce B-cell lymphoma in mice, LUBAC overexpression promoted B-lymphomagenesis induced by oncogenic mutation of MyD88. ${ }^{54)}$ Further analyses revealed that enforced LUBAC activity causes lymphomagenesis by preventing cell death related to activation-induced cytidine deaminase-induced DNA damage as well as by increasing $\mathrm{NF}-\kappa \mathrm{B}$ signaling. A natural compound that specifically inhibits LUBAC suppresses the growth of B-lymphomas in a mouse transplantation model. ${ }^{54)}$

In addition to B-cell lymphomas, increased LUBAC activity is involved in the resistance to cancer therapies. LUBAC plays a role in the resistance to a widely used anti-cancer drug, cisplatinum, in squamous cell lung cancer. ${ }^{86), 87)}$ LUBAC is also involved in resistance to anti-programmed death-1 therapy in murine B16F10 melanoma cells. ${ }^{88), 89)}$ Thus, inhibition of LUBAC may be a promising therapeutic strategy for a broad spectrum of malignant tumors.

c) Infectious diseases. LUBAC is involved in the clearance of intracellular pathogens (Fig. 4). Upon invasion into cells, Salmonella evades phagosomes. LUBAC is recruited to the intracellular Salmonella and coats the bacteria with linear ubiquitin. This induces NF- $\kappa \mathrm{B}$ activation on site and recruits the autophagic machinery, which delivers the pathogens to a destructive compartment via xenophagy. ${ }^{62), 63)}$ Several intracellular pathogens secretes toxins that inhibit linear ubiquitination. Legionella secretes a DUB that specifically cleaves

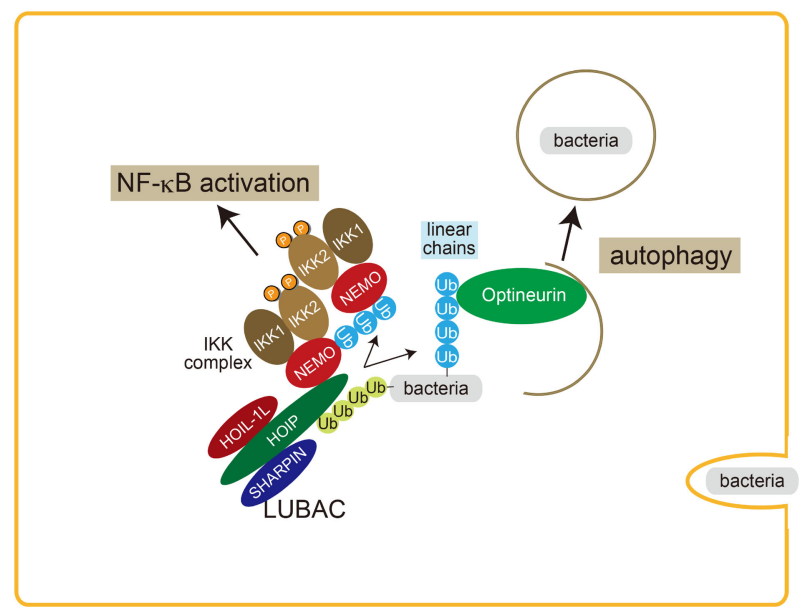

Fig. 4. (Color online) Mechanism underlying clearance of intracellular pathogens by LUBAC. Upon invasion into cells, bacteria evade phagosomes. LUBAC is recruited to the intracellular pathogens and coats them with linear ubiquitin, which induces $\mathrm{NF}-\kappa \mathrm{B}$ activation on site and clears pathogens via autophagy.

linear ubiquitin and dampens LUBAC-mediated signaling on the surface of bacteria. ${ }^{90)}$ Some effectors of Shigella are ubiquitin ligases that cause ubiquitinmediated degradation of HOIP to inhibit linear ubiquitination. ${ }^{91)}$ Gliotoxin, a critical virulence factor of the opportunistic pathogen Aspergillus, inhibits the linear ubiquitination activity of HOIP. ${ }^{92)}$ LUBAC is also involved in the clearance of several viruses including norovirus. ${ }^{69)}$ Taken together, these studies indicate that LUBAC-mediated linear ubiquitination is involved in protection against pathogen infection.

d) Neuromuscular diseases. Although mutations of LUBAC subunits underlie immunological diseases, a substantial number of patients with mutations in HOIL-1L exhibit myopathies, including cardiomyopathy with polyglucosan bodies; the precise molecular mechanism underlying polyglucosan body myopathy remains to be clarified. ${ }^{93)}$ In patients lacking immunological symptoms, the mutations are often located in the C-terminal half of HOIL-1L. ${ }^{94)}$ HOIL-1L interacts with HOIP and SHARPIN at the N-terminal region, ${ }^{14), 15)}$ and patients with mutations in the C-terminal half have substantial amounts of LUBAC, which explains the lack of immunological symptoms. Optineurin is one of the causative gene products of the motor neuron disease, amyotrophic lateral sclerosis. ${ }^{64)}$ Although mutations in Optineurin cause NF- $\kappa \mathrm{B}$ activation or impair autophagy, the precise mechanism leading to motor neuron disease remains unclear. 


\section{Manipulation of LUBAC activity}

The role of increased LUBAC activity in oncogenesis and resistance to anti-cancer treatment, as well as in some autoimmune diseases, has promoted interest in developing LUBAC inhibitors. Gliotoxin, a fungal metabolite, was the first small molecule shown to inhibit linear ubiquitination activity. ${ }^{92)}$ Thiolutin and aureotricin, products of Streptomycetes, also inhibit linear ubiquitination activity. ${ }^{54)}$ Although these compounds inhibit the growth of B-lymphoma cells in vitro or in mice, they are not specific to LUBAC. HOIPIN-8 is a synthetic agent that inhibits LUBAC linear ubiquitination by interacting specifically with HOIP. ${ }^{95)}$ Because loss of LUBAC activity causes embryonic lethality, ${ }^{15), 43)}$ the generation of molecules that decrease LUBAC activity has been attempted. Considering the critical roles of LTM-mediated dimerization of HOIL-1L and SHARPIN, ${ }^{15)}$ a stable peptide that specifically inhibits the interaction between the LTMs of HOIL$1 \mathrm{~L}$ and SHARPIN would decrease the amount of LUBAC and inhibit the growth of B-lymphoma cells.

Attenuated LUBAC activity underlies certain autoinflammatory conditions and pathogen infection. Loss of the ligase activity of HOIL-1L suppresses auto-linear ubiquitination of LUBAC; therefore, loss of the HOIL-1L E3 activity ameliorates dermatitis in cpdm mice caused by attenuated LUBAC activity and protects hepatocytes from apoptosis in mice. ${ }^{18)}$ Loss of the HOIL-1L E3 also protects cells from Salmonella infection. Because mice lacking the HOIL1L E3 do not show any overt phenotypes, ${ }^{18)}$ inhibitors of HOIL-1L may be of value for the treatment of infectious diseases and immune-compromised conditions.

\section{Final remarks}

This review summarized the current status of linear ubiquitin research. To conclude this review, the events leading to the discovery of linear ubiquitin chains and LUBAC can be examined. This research was initiated by a simple question: "How are ubiquitin chains generated via the repetitive functions of three enzymes?"96) Ubiquitin chains are conjugated to substrates recognized by E3s through the repetitive function of three enzymes, E1, E2, and E3. In the first reaction, ubiquitin is conjugated to a $\mathrm{K}$ residue in the substrate, which is followed by conjugation of ubiquitin to a $\mathrm{K}$ residue of ubiquitin moiety conjugated to proteins. ${ }^{2)}$ Because E3s recognize substrates, the spatial relationship between

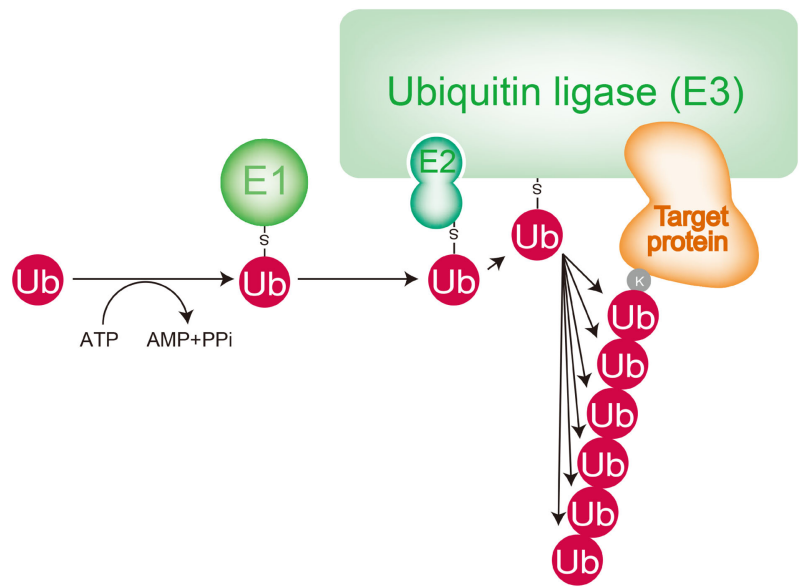

Fig. 5. (Color online) Proposed mechanism for the generation of ubiquitin chains at the time of discovery of linear ubiquitination. The repetitive activity of three enzymes, E1, E2, and E3, leads to the conjugation of ubiquitin to substrates recognized by E3s. Ubiquitin is first conjugated onto a substrate, followed by the conjugation of ubiquitin moieties onto the distal end of the ubiquitin chain. Because E3s recognize substrates, the spatial relation between enzymes (E3s) and reaction sites (interubiquitin linkage) changes during the chain elongation reaction.

enzymes (E3s) and the reaction sites (inter-ubiquitin linkage) changes during the chain elongation step (Fig. 5). ${ }^{96)}$ This phenomenon contradicts the principles of enzymology because enzymes facilitate reactions by recognizing a substrate through its catalytic site to reduce the energy of reaction intermediates.

During the study of the regulation of mammalian iron metabolism, HOIL-1 was identified by the author as a candidate E3 for the iron-dependent degradation of IRP2, a master regulator of cellular iron metabolism. ${ }^{97)} \mathrm{A}$ database search identified a longer isoform, HOIL-1L. The difference between the two isoforms was investigated using gel filtration analyses, which showed that HOIL-1L, but not HOIL-1, forms a high-molecular weight complex of approximately $\left.600 \mathrm{kDa} .{ }^{8}\right)$ Because HOIL-1L is the major isoform of HOIL-1, we focused on investigating the high-molecular weight complex, which led to the identification of HOIP as a HOIL-1L interactor. Although we later identified SHARPIN as the third component of $\mathrm{LUBAC},{ }^{11)}$ we failed to identify it during the purification of HOIL-1L interacting proteins possibly because SHARPIN co-electrophoresed with $\beta$-actin. As illustrated in Fig. 1, in addition to two E3 ligase centers, the complex has multiple domains. Among these, the ubiquitin-associated and NZF domains are ubiquitin-binding domains. We then hypothesized that this ligase complex 
recognizes ubiquitin and conjugates ubiquitin onto the recognized ubiquitin to form ubiquitin chains. This would imply that the reaction site does not change during the chain elongation reaction because the complex preferably recognizes the distal ubiquitin moieties of chains. Experiments performed to confirm the hypothesis led to the identification of a new type of ubiquitin chain and the LUBAC ubiquitin ligase complex.

The role of LUBAC-mediated linear ubiquitination in immune signaling and oncogenesis is wellestablished. Impairment or overactivation of linear ubiquitination causes autoinflammation or malignancy, respectively, and numerous pathogens infect organisms by targeting linear ubiquitination. Thus, LUBAC is a suitable target for the treatment of infections, immunological diseases, and cancer. Our studies provide unique strategies to inhibit as well as promote LUBAC-mediated linear ubiquitination, and it is expected that the discovery and elucidation of LUBAC-mediated linear ubiquitination will further contribute to human welfare.

\section{Acknowledgments}

The author is grateful to members of the Iwai laboratory for valuable discussions and insightful comments. Work in the Iwai laboratory is partly supported by grants from the Ministry of Education, Culture, Sports, Science, and Technology of Japan (Grant Numbers: 24112002, JP17H06174, and JP18H05499) and the Takeda Foundation (to K.I.).

\section{References}

1) Creasy, D.M. and Cottrell, J.S. (2004) Unimod: Protein modifications for mass spectrometry. Proteomics 4, 1534-1536.

2) Hershko, A. and Ciechanover, A. (1998) The ubiquitin system. Annu. Rev. Biochem. 67, 425479.

3) Ciechanover, A., Hod, Y. and Hershko, A. (1978) A heat-stable polypeptide component of an ATPdependent proteolytic system from reticulocytes. Biochem. Biophys. Res. Commun. 81, 1100-1105.

4) Komander, D. and Rape, M. (2012) The ubiquitin code. Annu. Rev. Biochem. 81, 203-229.

5) Chau, V., Tobias, J.W., Bachmair, A., Marriott, D., Ecker, D.J., Gonda, D.K. et al. (1989) A multiubiquitin chain is confined to specific lysine in a targeted short-lived protein. Science 243, 15761583.

6) Spence, J., Sadis, S., Haas, A.L. and Finley, D. (1995) A ubiquitin mutant with specific defects in DNA repair and multiubiquitination. Mol. Cell. Biol. 15, 1265-1273.

7) Peng, J., Schwartz, D., Elias, J.E., Thoreen, C.C.,
Cheng, D., Marsischky, G. et al. (2003) A proteomics approach to understanding protein ubiquitination. Nat. Biotechnol. 21, 921-926.

8) Kirisako, T., Kamei, K., Murata, S., Kato, M., Fukumoto, H., Kanie, M. et al. (2006) A ubiquitin ligase complex assembles linear polyubiquitin chains. EMBO J. 25, 4877-4887.

9) Gerlach, B., Cordier, S.M., Schmukle, A.C., Emmerich, C.H., Rieser, E., Haas, T.L. et al. (2011) Linear ubiquitination prevents inflammation and regulates immune signalling. Nature $\mathbf{4 7 1}$, 591-596.

10) Ikeda, F., Deribe, Y.L., Skanland, S.S., Stieglitz, B., Grabbe, C., Franz-Wachtel, M. et al. (2011) SHARPIN forms a linear ubiquitin ligase complex regulating $\mathrm{NF}-\kappa \mathrm{B}$ activity and apoptosis. Nature 471, 637-641.

11) Tokunaga, F., Nakagawa, T., Nakahara, M., Saeki, Y., Taniguchi, M., Sakata, S. et al. (2011) SHARPIN is a component of the NF- $\kappa$ B-activating linear ubiquitin chain assembly complex. Nature 471, 633-636.

12) Smit, J.J., Monteferrario, D., Noordermeer, S.M., van Dijk, W.J., van der Reijden, B.A. and Sixma, T.K. (2012) The E3 ligase HOIP specifies linear ubiquitin chain assembly through its RING-IBRRING domain and the unique LDD extension. EMBO J. 31, 3833-3844.

13) Stieglitz, B., Rana, R.R., Koliopoulos, M.G., MorrisDavies, A.C., Schaeffer, V., Christodoulou, E. et al. (2013) Structural basis for ligase-specific conjugation of linear ubiquitin chains by HOIP. Nature 503, 422-426.

14) Iwai, K., Fujita, H. and Sasaki, Y. (2014) Linear ubiquitin chains: NF- $\kappa$ B signalling, cell death and beyond. Nat. Rev. Mol. Cell Biol. 15, 503-508.

15) Fujita, H., Tokunaga, A., Shimizu, S., Whiting, A.L., Aguilar-Alonso, F., Takagi, K. et al. (2018) Cooperative domain formation by homologous motifs in HOIL-1L and SHARPIN plays a crucial role in LUBAC stabilization. Cell Rep. 23, 11921204.

16) Smit, J.J., van Dijk, W.J., El Atmioui, D., Merkx, R., Ovaa, H. and Sixma, T.K. (2013) Target specificity of the E3 ligase LUBAC for ubiquitin and NEMO relies on different minimal requirements. J. Biol. Chem. 288, 31728-31737.

17) Kelsall, I.R., Zhang, J., Knebel, A., Arthur, J.S.C. and Cohen, P. (2019) The E3 ligase HOIL-1 catalyses ester bond formation between ubiquitin and components of the Myddosome in mammalian cells. Proc. Natl. Acad. Sci. U.S.A. 116, 1329313298

18) Fuseya, Y., Fujita, H., Kim, M., Ohtake, F., Nishide, A., Sasaki, K. et al. (2020) The HOIL-1L ligase modulates immune signalling and cell death via monoubiquitination of LUBAC. Nat. Cell Biol. 22, 663-673.

19) Lork, M., Verhelst, K. and Beyaert, R. (2017) CYLD, A20 and OTULIN deubiquitinases in NF$\kappa \mathrm{B}$ signaling and cell death: So similar, yet so different. Cell Death Differ. 24, 1172-1183. 
20) Takiuchi, T., Nakagawa, T., Tamiya, H., Fujita, H., Sasaki, Y., Saeki, Y. et al. (2014) Suppression of LUBAC-mediated linear ubiquitination by a specific interaction between LUBAC and the deubiquitinases CYLD and OTULIN. Genes Cells 19, 254-272.

21) Elliott, P.R., Leske, D., Hrdinka, M., Bagola, K., Fiil, B.K., McLaughlin, S.H. et al. (2016) SPATA2 links CYLD to LUBAC, activates CYLD, and controls LUBAC signaling. Mol. Cell 63, 9901005.

22) Feltham, R., Webb, A.I. and Silke, J. (2016) SPATA2 - Keeping the TNF signal short and sweet. EMBO J. 35, 1848-1850.

23) Kupka, S., De Miguel, D., Draber, P., Martino, L., Surinova, S., Rittinger, K. et al. (2016) SPATA2mediated binding of CYLD to HOIP enables CYLD recruitment to signaling complexes. Cell Rep. 16, 2271-2280.

24) Bignell, G.R., Warren, W., Seal, S., Takahashi, M., Rapley, E., Barfoot, R. et al. (2000) Identification of the familial cylindromatosis tumour-suppressor gene. Nat. Genet. 25, 160-165.

25) Sun, S.C. (2010) CYLD: A tumor suppressor deubiquitinase regulating $\mathrm{NF}-\kappa \mathrm{B}$ activation and diverse biological processes. Cell Death Differ. 17, $25-34$.

26) Damgaard, R.B., Walker, J.A., Marco-Casanova, P., Morgan, N.V., Titheradge, H.L., Elliott, P.R. et al. (2016) The deubiquitinase OTULIN is an essential negative regulator of inflammation and autoimmunity. Cell 166, 1215-1230.E20.

27) Heger, K., Wickliffe, K.E., Ndoja, A., Zhang, J., Murthy, A., Dugger, D.L. et al. (2018) OTULIN limits cell death and inflammation by deubiquitinating LUBAC. Nature 559, 120-124.

28) Cohen, P. and Strickson, S. (2017) The role of hybrid ubiquitin chains in the MyD88 and other innate immune signalling pathways. Cell Death Differ. 24, 1153-1159.

29) Haakonsen, D.L. and Rape, M. (2019) Branching out: Improved signaling by heterotypic ubiquitin chains. Trends Cell Biol. 29, 704-716.

30) Emmerich, C.H., Ordureau, A., Strickson, S., Arthur, J.S., Pedrioli, P.G., Komander, D. et al. (2013) Activation of the canonical IKK complex by K63/M1-linked hybrid ubiquitin chains. Proc. Natl. Acad. Sci. U.S.A. 110, 15247-15252.

31) Fennell, L.M., Rahighi, S. and Ikeda, F. (2018) Linear ubiquitin chain-binding domains. FEBS J. 285, 2746-2761.

32) Sato, Y., Fujita, H., Yoshikawa, A., Yamashita, M., Yamagata, A., Kaiser, S.E. et al. (2011) Specific recognition of linear ubiquitin chains by the Npl4 zinc finger (NZF) domain of the HOIL-1L subunit of the linear ubiquitin chain assembly complex. Proc. Natl. Acad. Sci. U.S.A. 108, 20520-20525.

33) Tokunaga, F., Nishimasu, H., Ishitani, R., Goto, E., Noguchi, T., Mio, K. et al. (2012) Specific recognition of linear polyubiquitin by A20 zinc finger 7 is involved in NF- $\kappa$ B regulation. EMBO J. 31, 3856-3870.
34) Verhelst, K., Carpentier, I., Kreike, M., Meloni, L., Verstrepen, L., Kensche, T. et al. (2012) A20 inhibits LUBAC-mediated NF- $\kappa$ B activation by binding linear polyubiquitin chains via its zinc finger 7. EMBO J. 31, 3845-3855.

35) Tokunaga, F., Sakata, S., Saeki, Y., Satomi, Y., Kirisako, T., Kamei, K. et al. (2009) Involvement of linear polyubiquitylation of NEMO in NF- $\kappa \mathrm{B}$ activation. Nat. Cell Biol. 11, 123-132.

36) Iwai, K. (2012) Diverse ubiquitin signaling in NF- $\kappa \mathrm{B}$ activation. Trends Cell Biol. 22, 355-364.

37) Ghosh, S. and Hayden, M.S. (2008) New regulators of NF- $\kappa \mathrm{B}$ in inflammation. Nat. Rev. Immunol. 8, 837-848.

38) Fujita, H., Rahighi, S., Akita, M., Kato, R., Sasaki, Y., Wakatsuki, S. et al. (2014) Mechanism underlying $\mathrm{I} \kappa \mathrm{B}$ kinase activation mediated by the linear ubiquitin chain assembly complex. Mol. Cell. Biol. 34, 1322-1335.

39) Shimizu, S., Fujita, H., Sasaki, Y., Tsuruyama, T., Fukuda, K. and Iwai, K. (2016) Differential involvement of the Npl4 zinc finger domains of SHARPIN and HOIL-1L in linear ubiquitin chain assembly complex-mediated cell death protection. Mol. Cell. Biol. 36, 1569-1583.

40) Lo, Y.C., Lin, S.C., Rospigliosi, C.C., Conze, D.B., Wu, C.J., Ashwell, J.D. et al. (2009) Structural basis for recognition of diubiquitins by NEMO. Mol. Cell 33, 602-615.

41) Rahighi, S., Ikeda, F., Kawasaki, M., Akutsu, M., Suzuki, N., Kato, R. et al. (2009) Specific recognition of linear ubiquitin chains by NEMO is important for NF- $\kappa$ B activation. Cell 136, 10981109.

42) Sasaki, Y., Sano, S., Nakahara, M., Murata, S., Kometani, K., Aiba, Y. et al. (2013) Defective immune responses in mice lacking LUBAC-mediated linear ubiquitination in B cells. EMBO J. 32, 2463-2476.

43) Peltzer, N., Rieser, E., Taraborrelli, L., Draber, P., Darding, M., Pernaute, B. et al. (2014) HOIP deficiency causes embryonic lethality by aberrant TNFR1-mediated endothelial cell death. Cell Rep. 9, 153-165.

44) Opipari, A.W. Jr., Boguski, M.S. and Dixit, V.M. (1990) The A20 cDNA induced by tumor necrosis factor alpha encodes a novel type of zinc finger protein. J. Biol. Chem. 265, 14705-14708.

45) Damgaard, R.B., Nachbur, U., Yabal, M., Wong, W.W., Fiil, B.K., Kastirr, M. et al. (2012) The ubiquitin ligase XIAP recruits LUBAC for NOD2 signaling in inflammation and innate immunity. Mol. Cell 46, 746-758.

46) Takeda, K. and Akira, S. (2004) TLR signaling pathways. Semin. Immunol. 16, 3-9.

47) Dubois, S.M., Alexia, C., Wu, Y., Leclair, H.M., Leveau, C., Schol, E. et al. (2014) A catalyticindependent role for the LUBAC in NF- $\kappa$ B activation upon antigen receptor engagement and in lymphoma cells. Blood 123, 2199-2203.

48) Sasaki, K., Himeno, A., Nakagawa, T., Sasaki, Y., Kiyonari, H. and Iwai, K. (2019) Modulation of 
autoimmune pathogenesis by $\mathrm{T}$ cell-triggered inflammatory cell death. Nat. Commun. 10, 3878.

49) Juilland, M. and Thome, M. (2018) Holding All the CARDs: How MALT1 Controls CARMA/CARDDependent Signaling. Front. Immunol. 9, 1927.

50) Yang, Y.K., Yang, C., Chan, W., Wang, Z., Deibel, K.E. and Pomerantz, J.L. (2016) Molecular determinants of scaffold-induced linear ubiquitinylation of B cell lymphoma/leukemia 10 (Bcl10) during T cell receptor and oncogenic caspase recruitment domain-containing protein 11 (CARD11) Signaling. J. Biol. Chem. 291, 25921-25936.

51) Christofferson, D.E., Li, Y. and Yuan, J. (2014) Control of life-or-death decisions by RIP1 kinase. Annu. Rev. Physiol. 76, 129-150.

52) Peltzer, N., Darding, M. and Walczak, H. (2016) Holding RIPK1 on the ubiquitin leash in TNFR1 signaling. Trends Cell Biol. 26, 445-461.

53) Niu, J., Shi, Y., Iwai, K. and Wu, Z.H. (2011) LUBAC regulates $\mathrm{NF}-\kappa \mathrm{B}$ activation upon genotoxic stress by promoting linear ubiquitination of NEMO. EMBO J. 30, 3741-3753.

54) Jo, T., Nishikori, M., Kogure, Y., Arima, H., Sasaki, K., Sasaki, Y. et al. (2020) LUBAC accelerates Bcell lymphomagenesis by conferring resistance to genotoxic stress on B cells. Blood 136, 684-697.

55) Asaoka, T., Almagro, J., Ehrhardt, C., Tsai, I., Schleiffer, A., Deszcz, L. et al. (2016) Linear ubiquitination by LUBEL has a role in Drosophila heat stress response. EMBO Rep. 17, 1624-1640.

56) Damgaard, R.B. and Pruneda, J.N. (2019) Legionella dismantles linear ubiquitin. Nat. Microbiol. 4, 1244-1245.

57) Baghdiguian, S., Martinand-Mari, C. and Mangeat, P. (2007) Using Ciona to study developmental programmed cell death. Semin. Cancer Biol. 17, $147-153$.

58) Kesavardhana, S., Malireddi, R.K.S. and Kanneganti, T.D. (2020) Caspases in cell death, inflammation, and pyroptosis. Annu. Rev. Immunol. 38, 567-595.

59) Rodgers, M.A., Bowman, J.W., Fujita, H., Orazio, N., Shi, M., Liang, Q. et al. (2014) The linear ubiquitin assembly complex (LUBAC) is essential for NLRP3 inflammasome activation. J. Exp. Med. 211, 1333-1347.

60) Dikic, I. (2017) Proteasomal and autophagic degradation systems. Annu. Rev. Biochem. 86, 193-224.

61) Li, F., Xu, D., Wang, Y., Zhou, Z., Liu, J., Hu, S. et al. (2018) Structural insights into the ubiquitin recognition by OPTN (optineurin) and its regulation by TBK1-mediated phosphorylation. Autophagy 14, 66-79.

62) Noad, J., von der Malsburg, A., Pathe, C., Michel, M.A., Komander, D. and Randow, F. (2017) LUBAC-synthesized linear ubiquitin chains restrict cytosol-invading bacteria by activating autophagy and NF- $\kappa$ B. Nat. Microbiol. 2, 17063.

63) van Wijk, S.J.L., Fricke, F., Herhaus, L., Gupta, J., Hotte, K., Pampaloni, F. et al. (2017) Linear ubiquitination of cytosolic Salmonella Typhimurium activates $\mathrm{NF}-\kappa \mathrm{B}$ and restricts bacterial proliferation. Nat. Microbiol. 2, 17066.

64) Maruyama, H., Morino, H., Ito, H., Izumi, Y., Kato, H., Watanabe, Y. et al. (2010) Mutations of optineurin in amyotrophic lateral sclerosis. Nature 465, 223-226.

65) Wild, P., Farhan, H., McEwan, D.G., Wagner, S., Rogov, V.V., Brady, N.R. et al. (2011) Phosphorylation of the autophagy receptor optineurin restricts Salmonella growth. Science 333, 228-233.

66) Yamano, K., Kikuchi, R., Kojima, W., Hayashida, R., Koyano, F., Kawawaki, J. et al. (2020) Critical role of mitochondrial ubiquitination and the OPTN-ATG9A axis in mitophagy. J. Cell Biol. 219, e201912144.

67) Heaton, S.M., Borg, N.A. and Dixit, V.M. (2016) Ubiquitin in the activation and attenuation of innate antiviral immunity. J. Exp. Med. 213, 113.

68) Inn, K.S., Gack, M.U., Tokunaga, F., Shi, M., Wong, L.Y., Iwai, K. et al. (2011) Linear ubiquitin assembly complex negatively regulates RIG-Iand TRIM25-mediated type I interferon induction. Mol. Cell 41, 354-365.

69) MacDuff, D.A., Baldridge, M.T., Qaqish, A.M., Nice, T.J., Darbandi, A.D., Hartley, V.L. et al. (2018) HOIL1 is essential for the induction of type I and III interferons by MDA5 and regulates persistent murine norovirus infection. J. Virol. 92, e01368-18.

70) Ben-Chetrit, E., Gattorno, M., Gul, A., Kastner, D.L., Lachmann, H.J., Touitou, I. et al. (2018) Consensus proposal for taxonomy and definition of the autoinflammatory diseases (AIDs): A Delphi study. Ann. Rheum. Dis. 77, 1558-1565.

71) HogenEsch, H., Gijbels, M.J., Offerman, E., van Hooft, J., van Bekkum, D.W. and Zurcher, C. (1993) A spontaneous mutation characterized by chronic proliferative dermatitis in C57BL mice. Am. J. Pathol. 143, 972-982.

72) Seymour, R.E., Hasham, M.G., Cox, G.A., Shultz, L.D., Hogenesch, H., Roopenian, D.C. et al. (2007) Spontaneous mutations in the mouse Sharpin gene result in multiorgan inflammation, immune system dysregulation and dermatitis. Genes Immun. 8, 416-421.

73) Boisson, B., Laplantine, E., Prando, C., Giliani, S., Israelsson, E., Xu, Z. et al. (2012) Immunodeficiency, autoinflammation and amylopectinosis in humans with inherited HOIL-1 and LUBAC deficiency. Nat. Immunol. 13, 1178-1186.

74) Boisson, B., Laplantine, E., Dobbs, K., Cobat, A., Tarantino, N., Hazen, M. et al. (2015) Human HOIP and LUBAC deficiency underlies autoinflammation, immunodeficiency, amylopectinosis, and lymphangiectasia. J. Exp. Med. 212, 939-951.

75) Zhou, Q., Yu, X., Demirkaya, E., Deuitch, N., Stone, D., Tsai, W.L. et al. (2016) Biallelic hypomorphic mutations in a linear deubiquitinase define otulipenia, an early-onset autoinflammatory disease. Proc. Natl. Acad. Sci. U.S.A. 113, 10127-10132.

76) Keusekotten, K., Elliott, P.R., Glockner, L., Fiil, B.K., Damgaard, R.B., Kulathu, Y. et al. (2013) OTULIN antagonizes LUBAC signaling by specif- 
ically hydrolyzing Met1-linked polyubiquitin. Cell 153, 1312-1326.

77) Elliott, P.R., Nielsen, S.V., Marco-Casanova, P., Fiil, B.K., Keusekotten, K., Mailand, N. et al. (2014) Molecular Basis and Regulation of OTULINLUBAC Interaction. Mol. Cell 54, 335-348.

78) Schaeffer, V., Akutsu, M., Olma, M.H., Gomes, L.C., Kawasaki, M. and Dikic, I. (2014) Binding of OTULIN to the PUB Domain of HOIP Controls NF- $\kappa$ B Signaling. Mol. Cell 54, 349-361.

79) Adrianto, I., Wang, S., Wiley, G.B., Lessard, C.J., Kelly, J.A., Adler, A.J. et al. (2012) Association of two independent functional risk haplotypes in TNIP1 with systemic lupus erythematosus. Arthritis Rheum. 64, 3695-3705.

80) Herhaus, L., van den Bedem, H., Tang, S., Maslennikov, I., Wakatsuki, S., Dikic, I. et al. (2019) Molecular Recognition of M1-Linked Ubiquitin Chains by Native and Phosphorylated UBAN Domains. J. Mol. Biol. 431, 3146-3156.

81) Nanda, S.K., Venigalla, R.K., Ordureau, A. Patterson-Kane, J.C., Powell, D.W., Toth, R. et al. (2011) Polyubiquitin binding to ABIN1 is required to prevent autoimmunity. J. Exp. Med. 208, 12151228.

82) Kuriakose, J., Redecke, V., Guy, C., Zhou, J., Wu, R., Ippagunta, S.K. et al. (2019) Patrolling monocytes promote the pathogenesis of early lupus-like glomerulonephritis. J. Clin. Invest. 129, 2251-2265.

83) Nanda, S.K., Petrova, T., Marchesi, F., Gierlinski, M., Razsolkov, M., Lee, K.L. et al. (2019) Distinct signals and immune cells drive liver pathology and glomerulonephritis in ABIN1[D485N] mice. Life Sci. Alliance 2, e201900533.

84) Yang, Y., Schmitz, R., Mitala, J., Whiting, A., Xiao, W., Ceribelli, M. et al. (2014) Essential role of the linear ubiquitin chain assembly complex in lymphoma revealed by rare germline polymorphisms. Cancer Discov. 4, 480-493.

85) Young, R.M., Shaffer, A.L. 3rd, Phelan, J.D. and Staudt, L.M. (2015) B-cell receptor signaling in diffuse large B-cell lymphoma. Semin. Hematol. 52, 77-85.

86) Mackay, C., Carroll, E., Ibrahim, A.F., Garg, A., Inman, G.J., Hay, R.T. et al. (2014) E3 ubiquitin ligase HOIP attenuates apoptotic cell death induced by cisplatin. Cancer Res. 74, 2246-2257.

87) Ruiz, E.J., Diefenbacher, M.E., Nelson, J.K., Sancho, R., Pucci, F., Chakraborty, A. et al. (2019) LUBAC determines chemotherapy resistance in squamous cell lung cancer. J. Exp. Med. 216, 450-
465.

88) Manguso, R.T., Pope, H.W., Zimmer, M.D., Brown, F.D., Yates, K.B., Miller, B.C. et al. (2017) In vivo CRISPR screening identifies Ptpn2 as a cancer immunotherapy target. Nature 547, 413-418.

89) Pan, D., Kobayashi, A., Jiang, P., Ferrari de Andrade, L., Tay, R.E., Luoma, A.M. et al. (2018) A major chromatin regulator determines resistance of tumor cells to $\mathrm{T}$ cell-mediated killing. Science 359, 770-775.

90) de Jong, M.F., Liu, Z., Chen, D. and Alto, N.M. (2016) Shigella flexneri suppresses NF- $\kappa$ B activation by inhibiting linear ubiquitin chain ligation. Nat. Microbiol. 1, 16084.

91) Wan, M., Wang, X., Huang, C., Xu, D., Wang, Z., Zhou, Y. et al. (2019) A bacterial effector deubiquitinase specifically hydrolyses linear ubiquitin chains to inhibit host inflammatory signalling. Nat. Microbiol. 4, 1282-1293.

92) Sakamoto, H., Egashira, S., Saito, N., Kirisako, T., Miller, S., Sasaki, Y. et al. (2015) Gliotoxin suppresses NF- $\kappa \mathrm{B}$ activation by selectively inhibiting linear ubiquitin chain assembly complex (LUBAC). ACS Chem. Biol. 10, 675-681.

93) Nilsson, J., Schoser, B., Laforet, P., Kalev, O., Lindberg, C., Romero, N.B. et al. (2013) Polyglucosan body myopathy caused by defective ubiquitin ligase RBCK1. Ann. Neurol. 74, 914919.

94) Krenn, M., Salzer, E., Simonitsch-Klupp, I., Rath, J., Wagner, M., Haack, T.B. et al. (2018) Mutations outside the N-terminal part of RBCK1 may cause polyglucosan body myopathy with immunological dysfunction: Expanding the genotype-phenotype spectrum. J. Neurol. 265, 394-401.

95) Katsuya, K., Oikawa, D., Iio, K., Obika, S., Hori, Y., Urashima, T. et al. (2019) Small-molecule inhibitors of linear ubiquitin chain assembly complex (LUBAC), HOIPINs, suppress NF- $\kappa \mathrm{B}$ signaling. Biochem. Biophys. Res. Commun. 509, 700-706.

96) Iwai, K. (2020) Discovery of linear ubiquitination, a crucial regulator for immune signaling and cell death. FEBS J. https://doi.org/10.1111/febs.15471 (in press).

97) Yamanaka, K., Ishikawa, H., Megumi, Y., Tokunaga, F., Kanie, M., Rouault, T.A. et al. (2003) Identification of the ubiquitin-protein ligase that recognizes oxidized IRP2. Nat. Cell Biol. 5, $336-340$.

(Received Dec. 9, 2020; accepted Dec. 25, 2020) 


\section{Profile}

Kazuhiro Iwai was born (1959) and grew up in Kyoto. He graduated from the Faculty of Medicine, Kyoto University, and received his M.D. in 1985. Following 2 years of residency in internal medicine, he received his Ph.D. from Kyoto University in 1992 (Mentor: Dr. Hiroo Imura). He joined the laboratory of Drs. Tracey A. Rouault and Richard D. Klausner at the National Institutes of Health, U.S.A., in 1993, as a postdoctoral fellow. He joined the Department of Immunology and Cell Biology, Graduate School of Medicine, Kyoto University (Dr. Nagahiro Minato) in 1996. He was appointed as a Professor in the Graduate School of Medicine, Osaka City University, in 2001, and moved to the Graduate School of Frontier Biosciences and Graduate School of Medicine in Osaka University in 2008. He was appointed as a Professor in the Graduate School of

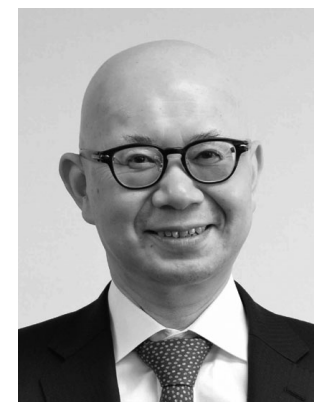
Medicine, Kyoto University, in 2012, and is currently the Dean of the Graduate School of Medicine, Kyoto University. His research focuses on the pathophysiology of LUBAC-mediated linear ubiquitination and iron metabolism. He has published papers on ubiquitin-mediated degradation of IRP2 and hypoxia-inducible factor $1 \alpha$; on the discovery of LUBAC-mediated linear ubiquitination, which plays crucial roles in immune-signaling; and on the pathophysiology of LUBAC-mediated linear ubiquitination. 